\title{
Compact 6+1 Antenna Array for Robust GNSS Applications
}

\author{
Stefano Caizzone, Wahid Elmarissi, Georg Buchner and Matteo Sgammini \\ Inst. of Communications and Navigation, German Aerospace Center (DLR) \\ Wessling, Germany - Email: stefano.caizzone@dlr.de
}

\begin{abstract}
The increase in GNSS satellites and services is fostering a new wave of applications related to satellite navigation. Such increase is also followed by more and more threats, aiming at signal disruption. In order to fully exploit the potentialities, being able at the same time to counteract threats such as interference and jamming, smart antenna systems are being developed worldwide, with the requirements of multi-band operation and compactness. In order to answer such need, the present work proposes a miniaturized $6+1$ array able to operate at E5, L2, E6 bands with the central element and at E1/L1 with 6 elements, providing therefore also beamforming and nulling capability at such band, with an overall footprint of only 7' (180 mm).

Index Terms-GNSS, array, miniaturization, DRA
\end{abstract}

\section{INTRODUCTION}

The development of new GNSS constellations and services alongside with the GPS system, as for instance the forthcoming european Galileo system, enables a multitude of novel applications for what concerns the precise positioning of people, vehicles and goods. Such increase of satellite navigation applications is however threatened by more and more interest in malicious attacks, for instance through jamming or spoofing, to deny the service [1] or even to deceive the user regarding the correctness of the navigation functionality [2].

In order to fully exploit the offered possibilities and at the same time counteract possible threats, high-performance systems, able to steer the antenna pattern and hence place nulls against interferences and maxima at the DOA of signals, are being developed all over the world, e.g. [3]. These new systems might be particularly useful in mobile applications, such as aircraft, vehicles or even drones and UAVs, where precise localization can play an important role. However, the canonical dimensions of the antennas, as well as of receivers, do not fit with the requirements of low-profile and low weight, typical of mobile applications: there is hence a need for miniaturization both of the single radiator and of the overall antenna array, to enable real-life implementation of the developed systems[8].

Moreover, benefits can be easily foreseen if antennas and receivers are able to process at the same time signals coming from multiple bands, with increases in precision, availability and robustness.

Various multiband GNSS arrays have been developed in the last years: in most cases, however, they have bulky dimensions (e.g. an overall diameter of $36.5 \mathrm{~cm}$ for a 7 element array as in [4]), due to the need for reducing mutual coupling between the elements and possibly also the use of commercial off the shelf (COTS) antennas as in [5]: such dimensions are however not yet suitable for integration in mobile platforms.

The present work shows a design for a miniaturized GNSS $6+1$ array (overall footprint dimension: 7 inches or equivalently $180 \mathrm{~mm}$ ) for operation as single antenna at E5/L2/E6 bands and as controlled reception pattern antenna (CRPA) at E1/L1: in such a way, the central antenna will provide reception for the lower navigation bands, helping in obtaining precise navigation, while the outer antennas will have the capability to suppress interferences in case of jammed scenarioes, hence enabling to obtain further navigation solutions also under jamming attacks. The single antennas are dielectric resonator antennas (DRAs) and are made of high dielectric constant (DK) bulk glass ceramic materials, enabling good miniaturization. The paper is divided as follows: Sec. II shows the single antenna design, while array design is explained in Sec. III. The interference suppression capability is experimented in Sec. IV. Finally, conclusions are drawn in Sec. V.

\section{Single Antenna Design}

Dielectric resonators (DRs) [6] have been thoroughfully analyzed in literature and used for various applications, beginning with microwave filters and oscillators. They have the characteristic property of having very few metal parts and hence exhibit quite low conductor losses even at very high frequency. Thanks to such feature, their use as antennas is now becoming popular (because low losses allow high radiation efficiency), also for GNSS applications [7], [8]. Their behaviour is based on the resonant mode(s) established in a dielectric material by a feeding element. Such mode will however not be totally confined into the dielectric material, but will also leak out, leading to radiation properties.

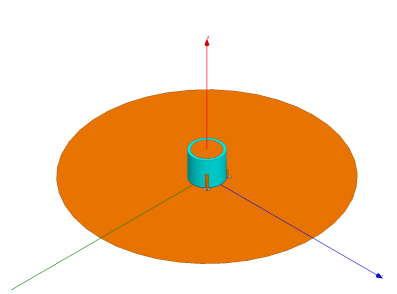

(a)

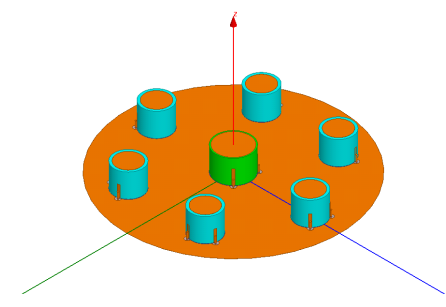

(b)
Figure 1. 3D view of the single DRA (a) and of the $6+1$ DRA array (b) on a circular ground plane (diameter: 7"). 
By using dielectric resonator antennas (DRAs) [9], three positive effects are generated: on the one hand, they usually make use of very high DK dielectric materials, hence enabling strong miniaturization, as the resonant frequency depends from the dimension and the DK of the DRA. On the other hand, they are not planar, but volume structures and hence give designers additional degrees of freedom during the design phase as well as allow for larger bandwidths. Finally, the fields are confined in their vicinity, so that improvements in mutual coupling behaviour for close-by elements are experienced.

In the present work, a bulk glass ceramic material with a DK of about 30 has been taken in consideration for the DRA. The antenna has a cylindrical shape and is placed on a 3.5" metallic plate. The feeding is obtained by means of two probes, tangential to the DRA surface on two $90^{\circ}$-shifted positions, to excite RHCP fields by means of an external $90^{\circ}$ hybrid circuit. The cylindrical probes are soldered to a copper strip, conformal to the cylinder, acting as tuning element. Moreover, the upper surface of the cylinder is partially covered by copper, helping in further miniaturization [8] (Fig. 1 (a)), as well as providing a further degree of freedom for tuning.

The single antenna element has a diameter of $23.5 \mathrm{~mm}$ (when operating at L1 band) or $29 \mathrm{~mm}$ (for E5/L2/E6 bands).
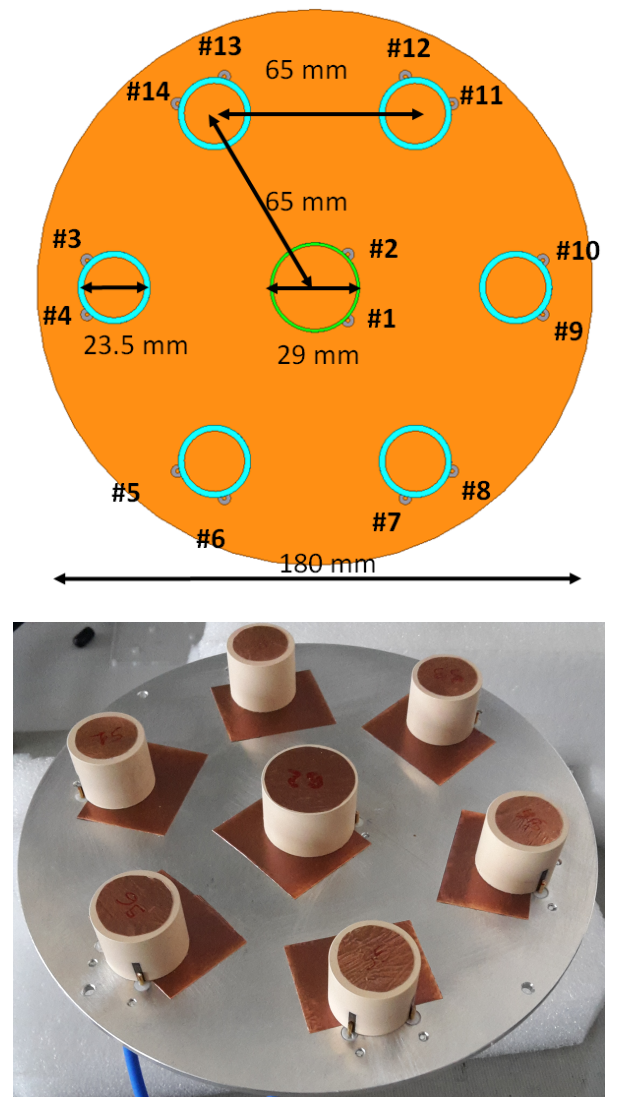

Figure 2. Scheme of the array with dimensions and port numbering (up) and manufactured array (down)

\section{ARray DESIGN AND MEASUREMENT}

The single antennas were then placed in a $6+1$ array configuration, as shown in Fig. 2.
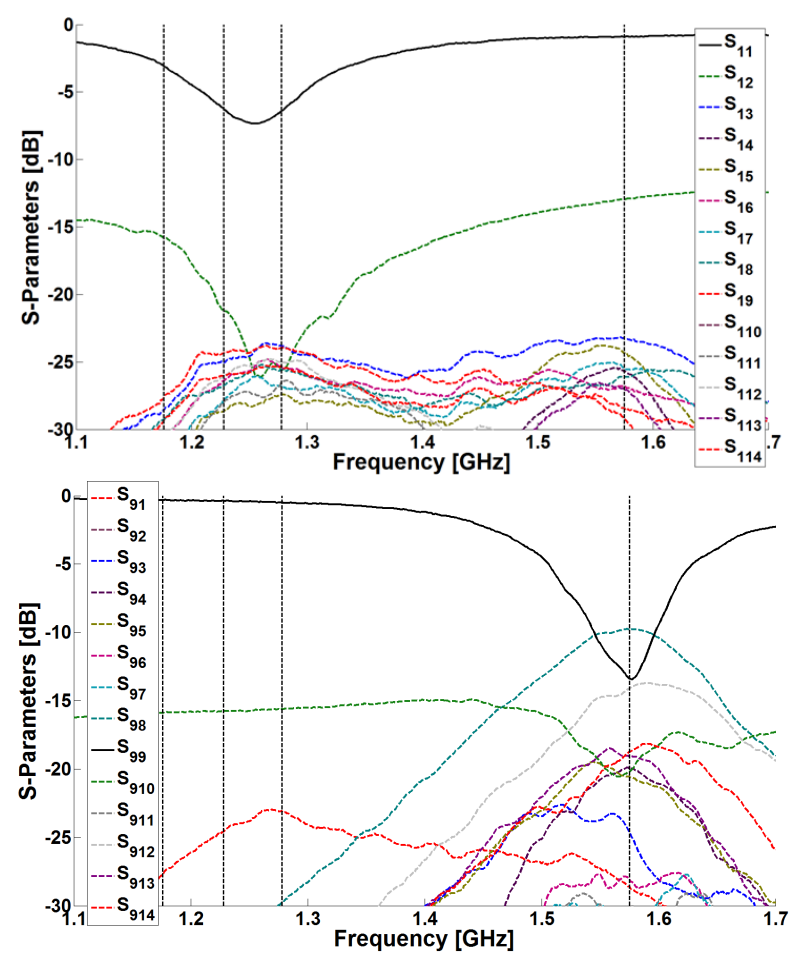

Figure 3. Measured S-Parameters for the central antenna (up) and for the outer antennas (down) . For port numbering, refer to Fig. 2
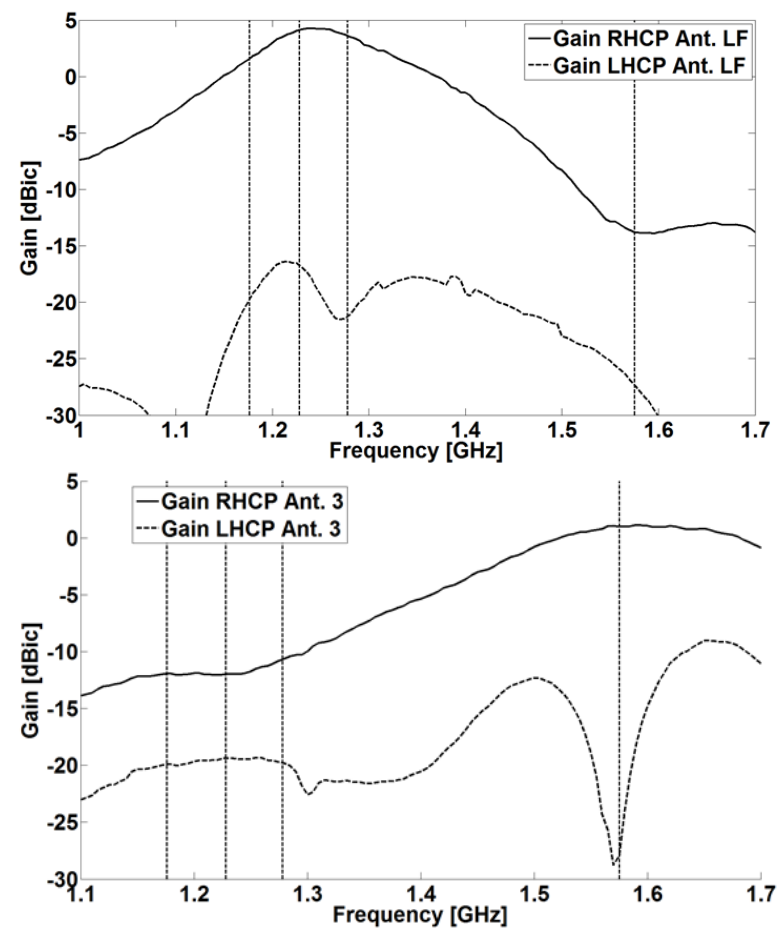

Figure 4. Measured embedded gain at boresight versus frequency for the central antenna (up) and for one of the outer antennas - Ant. 3 (down) 
The 6 outer antennas were sequentially rotated to improve the polarization purity of the array [10] and the dimensions and tuning were optimized.

The antennas were then manufactured (Fig. 2-b) and measured: the S-Parameters were measured with a vector network analyzer (VNA) both for the central and for the outer antennas, as shown in Fig. 3.

Farfield properties were instead measured in a Satimo Starlab measurement facility: the gain at boresight versus frequency is shown in Fig. 4, while the 3D patterns at L2 and L1 respectively are reported in Fig. 5.

Good behaviour at both bands is visible: the central antenna has almost no coupling with the outer antenans, as shown in the very low $S_{1 k}$ curves in Fig. 3, hence effectively letting it operate as if in standalone configuration. The pattern of the central antenna is moreover very symmetric and well covering the whole upper hemisphere (Fig. 5-up). On the other hand, the outer antennas exhibit more coupling, but still all mutual S-Parameters are below -10 dB (Fig. 3-down), hence allowing for good performance. As a matter of fact, the pattern of the outer antennas, as expected, are slightly squinted, due to their non-symmetric placement on the ground plane, as well as because of the influence of the neighbouring antennas of the array. Nevertheless, a good gain coverage for the whole upper hemisphere is available and will help in obtaining the interference suppression capability for up to 5 interference directions, as shown in next Section.
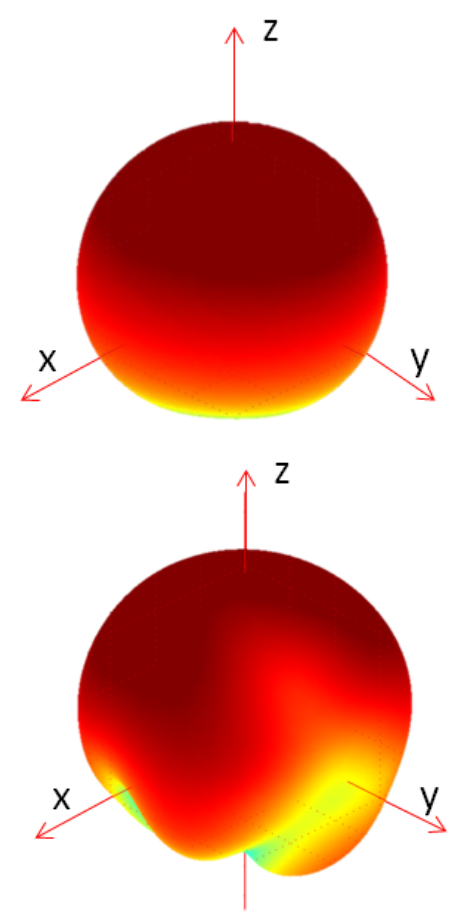

Figure 5. Measured 3D embedded gain for the central antenna at $1225 \mathrm{MHz}$ (up) and for one of the outer antennas at $1575 \mathrm{MHz}$ (down)

\section{INTERFERENCE SUPPRESSION CAPABILITY}

In order to simply check the interference suppression capability of the proposed antenna, a deterministic beamforming algorithm has been implemented [11].

After identifying different scenarioes and the respective interference directions, the optimal weights for each array element (in order to obtain nulls in the directions of interferers and good coverage of the other directions) were found by solving

$$
w=w_{D}\left(I-C\left[C^{\prime} C\right]^{-1} C^{\prime}\right)
$$

with $w_{D}$ being the steering vector in the wanted direction (here assumed as boresight), $I$ being the identity matrix and $C$ being a matrix containing the steering vectors in the directions of the nulls [11].
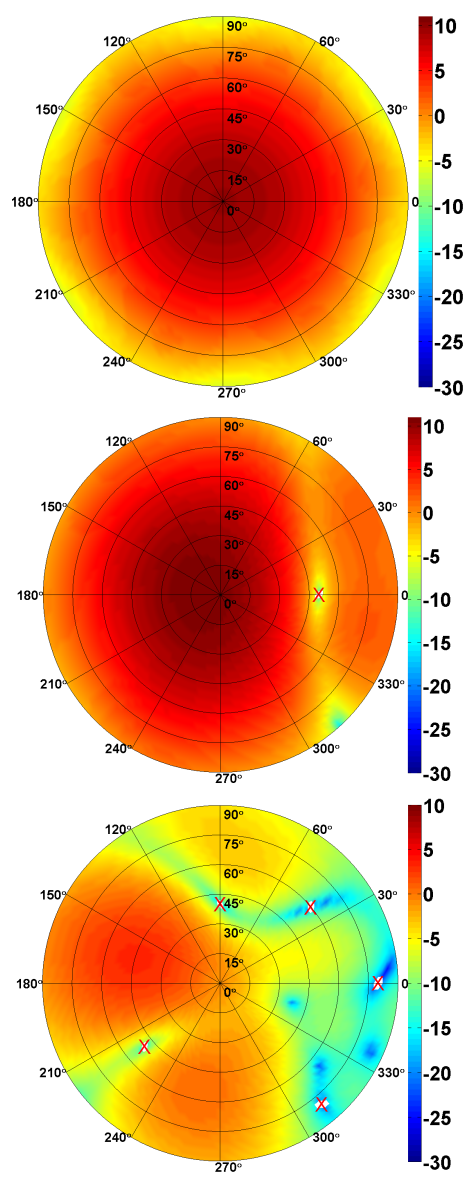

Figure 6. Simulated 3D array gain in $\mathrm{dBi}$ after nulling at $1575 \mathrm{MHz}$ for, respectively, interference-free case (top), 1 interferer case (middle), 5 interferers (bottom). Interference directions are marked with a red X.

Thanks to the 6 outer elements, it will theoretically be possible to place 5 (i.e. number of antennas-1) nulls in the overall array pattern.

In order to prove its nulling capability in some reference scenarioes, the array has been tested in simulation in the following cases: 
- interference-free case

- 1 interferer (coming from $\left.\{\theta, \varphi\}\left[^{\circ}\right]=\{[50,0]\}\right)$

- 5 interferers (coming from $\{\theta, \varphi\}\left[^{\circ}\right]=$ $\{[80,0] ;[60,40] ;[40,90] ;[50,220] ;[80,310]\}$

The resulting patterns after proper weighting of the embedded patterns through the optimal weighting vector are shown in Fig. 6: a clear capability of null placement is demonstrated, as well as a good coverage of the remaining part of the upper hemisphere.

Further results will be shown during the conference.

\section{CONCLUSIONS}

The present paper has shown the development and measurement of a compact $6+1$ controlled radiation pattern antenna array (CRPA) for robust GNSS navigation. Thanks to the use of miniaturized antennas, it was possible to have 7 antennas in a very small footprint of $180 \mathrm{~mm}$ (= 7 inches), hence obtaining a substantial improvement with regards to current CRPAs and therefore potentially enabling a broader use in mobile applications. Moreover, the use of one central antenna for the E5/L2/E6 bands permits to have a secundary navigation band capability, helping to obtain more precise navigation. The outer 6 elements, on the other hand, exhibit inferference suppression capability, as shown in the paper, and allow for further navigation also in moderately jammed scenarioes.

\section{REFERENCES}

[1] D. Borio et al., "Jammer Impact on Galileo and GPS Receivers", Proc of 2013 Int. Conf. on Localization and GNSS (ICL-GNSS), Torino (Italy), Jun.2013

[2] J.A. Larcom, Hong Liu, "Modeling and Characterization of GPS Spoofing", Proc. of 2013 Int. Conf. on Technologies for Homeland Security (HST), Waltham (USA), Nov. 2013

[3] M. V. T. Heckler et al. , "Development of robust safety-of-life navigation receivers", IEEE Trans. Microw. Theory Tech., vol. 59, no. 4, pp. 9981005, Apr. 2011

[4] F. Leveau et al., "Anti-Jam Protection by Antenna: Conception, Realization and Evaluation of a Seven-Element GNSS CRPS", GPS World, Feb. 2013

[5] Y.-H. Chen et al., "Off-the-Shelf Antennas for Controlled-ReceptionPattern Antenna Arrays",GPS World, Feb. 2013

[6] A. Sharma, S.C. Shrivastava, "Analysis of Resonant Frequency and Quality Factor of Dielectric Resonator at Different Dielectric Constant Materials", Proc. of 2008 Int. Conf. on Recent Advances in Microwave Theroy and App., Jaipur (India), Nov. 2008

[7] M. Clenet, M. Caillet, Y. M. M. Antar, "Wideband Circularly Polarized Antenna Elements for GPS/GNSS Applications Developed at DRDC Ottawa", Proc. of 2010 Int. Symp. on Antenna Tech. and Applied Electromagnetics (ANTEM 2010), Ottawa (Canada), Jul. 2010

[8] S. Caizzone, A. Dreher, "Miniaturized DRA Array for GNSS Applications", Proc. of 9th European Conf. on Antennas and Propag. (EUCAP 2015), Lisbon (Portugal), Apr. 2015

[9] A. Petosa, A. Ittipiboon, "Dielectric Resonator Antennas: a Historical Review and the Current State of the Art", IEEE Antennas Propag. Mag., vol. 52, no. 5, pp.91-116, Oct. 2010

[10] U. R. Kraft, "Main-Beam Polarization Properties of Four-Element Sequential-Rotation Arrays with Arbitrary Radiators", IEEE Trans. Antennas Propag., vol. 44, no. 4, pp. 515-522, Apr. 1996

[11] H. L. Van Trees, "Optimum Array Processing: Part IV of Detection, Estimation and Modulation Theory", Wiley-Interscience, New York (USA), 2002 